\title{
Theoretical study of Čerenkov-type second-harmonic generation in periodically poled ferroelectric crystals
}

\author{
Yan Sheng, ${ }^{1, *}$ Qian Kong, ${ }^{1,2}$ Vito Roppo, ${ }^{3}$ Ksawery Kalinowski, ${ }^{1}$ Qi Wang, ${ }^{2}$ \\ Crina Cojocaru, ${ }^{3}$ and Wieslaw Krolikowski ${ }^{1}$ \\ ${ }^{1}$ Laser Physics Center and Nonlinear Physics Center, Research School of Physics and Engineering, \\ Australian National University, Canberra ACT 0200, Australia \\ ${ }^{2}$ Department of Physics, Shanghai University, Shanghai 200444, China \\ ${ }^{3}$ Departament de Fisica i Enginyeria Nuclear, Universitat Politecnica de Catalunya, \\ Rambla Sant Nebridi, 08222 Terrassa, Barcelona, Spain \\ *Corresponding author: ysh111@physics.anu.edu.au
}

Received August 25, 2011; accepted November 7, 2011;

posted November 14, 2011 (Doc. ID 153486); published February 13, 2012

\begin{abstract}
We study theoretically the Čerenkov-type second-harmonic generation by a Gaussian beam in quadratic nonlinear media with periodically reverted ferroelectric domains. In particular, we consider the role of fundamental beam width on the harmonic emission process. We show that varying the beam width of the fundamental wave not only affects the strength of the emitted Čerenkov harmonic signal but also changes the sensitivity of the emission process to wavelength tuning. (C) 2012 Optical Society of America
\end{abstract}

OCIS codes: $\quad 190.2620,190.4410,220.4000$.

\section{INTRODUCTION}

Optical parametric processes that involve, for instance, second-harmonic ( $\mathrm{SH})$, sum, and difference frequency generation have been studied extensively since the conception of the lasers $[1,2]$. They are of great practical importance since they can be used to create coherent light sources at the frequency bands where the conventional lasers either perform poorly or are unavailable at all. It is well established that the efficiency of the parametric process critically depends on the matching of phase velocities of interacting waves. However, this is difficult to satisfy because the optical dispersion of crystals gives rise to different phase velocities of interacting light waves with different frequencies. Therefore, the efficient parametric process requires phase compensation of optical dispersion of the nonlinear medium. This has traditionally been done utilizing the birefringence of the crystals and temperature tuning [3]. A much more versatile technique involves the quasiphase-matching (QPM) [4] that uses periodically poled ferroelectric crystals [5-9]. In such crystals with antiparallel domains, the linear refractive index is spatially constant but the sign of certain components of the quadratic nonlinear susceptibility tensor $\chi^{(2)}$ varies periodically. The resulting modulation of the nonlinearity with a period $\Lambda$ can phase match interactions with a wave vector difference that equals integral multiples of $2 \pi / \Lambda$ over the transparency range of the nonlinear crystal. In the past years, the quasi-phase-matched nonlinear processes have been intensively studied with the fundamental beam propagating in a plane perpendicular to the domain walls [the boundaries between the positive and negative domains, see Fig. 1(a)].

Recently, efficient $\mathrm{SH}$ generation (SHG) in periodic $\chi^{(2)}$ crystals that satisfies only the longitudinal phase-matching condition, i.e., $k_{2} \cos \theta=2 k_{1}$ [10-15] was also demonstrated. Here $k_{1}$ and $k_{2}$ represent wave vectors of the fundamental and $\mathrm{SH}$ waves and $\theta$ is the angle between them. The longitudinally phase-matched SHG is also known as Čerenkov SHG because of the close analogy with the famous Čerenkov effect [16]. The nonlinear polarization at the doubled fundamental frequency, which propagates in a nonlinear medium with phase velocity $(\nu)$ greater than the speed of the optical $\mathrm{SH}$ wave $\left(\nu^{\prime}\right)$ in the same medium, can radiate the optical SH signal at an angle determined by $\cos \theta=\nu^{\prime} / \nu=2 k_{1} / k_{2}$. While several publications reported the observation of Čerenkov SHG in homogeneous bulk nonlinear crystal [17-19], it has been shown that this type of SHG is much more easily observed in periodic $\chi^{(2)}$ media with the fundamental beam propagating along the domain walls [see Figs. 1(b) and (c)]. The presence of $\chi^{(2)} \bmod -$ ulation can greatly enhance the harmonic emission intensity [20-22]. The exact physical origin of this enhancement is still unclear, and it is a subject of intense investigations that consider either the role of the reciprocal vectors in the nonlinear photonic structure [20] or enhancement of the quadratic nonlinearity on the domain walls [21,22].

While Čerenkov SHG has been studied in $\chi^{(2)}$ crystals for some time, the physical features of this type of interaction are still not fully understood. In the current study, we study analytically the Čerenkov SHG by a Gaussian beam in periodically poled $\mathrm{LiNbO}_{3}$ crystal. We demonstrate for the first time, to the best of our knowledge, that one needs to take the beam width of the fundamental wave into account when discussing the properties of the Čerenkov interaction as it dramatically affects the interaction. Our results clearly show that varying the beam width of the fundamental wave not only affects the strength of the emitted Čerenkov harmonic signal but also changes the sensitivity of the emission process to wavelength tuning.

\section{THEORETICAL MODEL AND EQUATIONS}

It is known that, with assumptions of weak focusing of the pump wave and its negligible depletion, the efficiency of SHG 


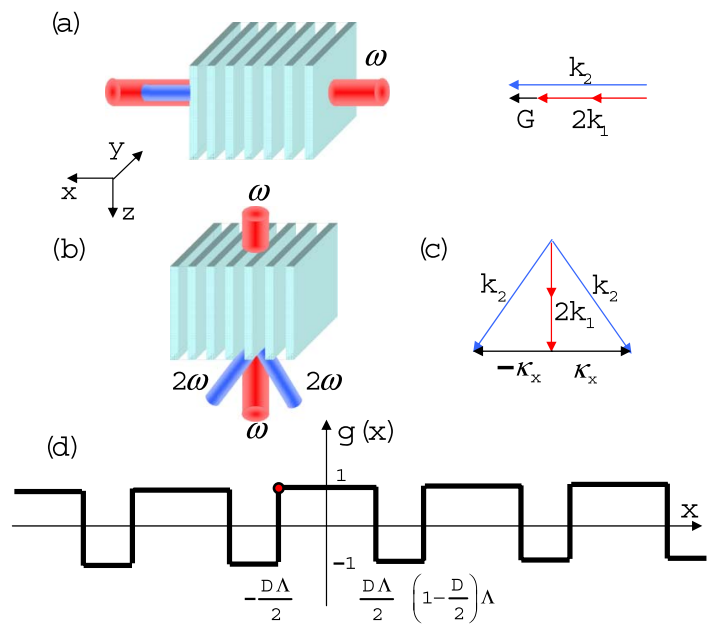

Fig. 1. (Color online) (a) Traditional quasi-phase-matched SHG with fundamental beam propagating along normal to the domain walls $(x$ axis). (b) Čerenkov-type SHG with fundamental wave directed along the domain walls ( $z$ axis). (c) Triangle constructed by the vectors $k_{2}, 2 k_{1}$, and $\kappa_{x}$ when the Čerenkov phase-matching condition is fulfilled, i.e., when the argument of the sinc function in Eq. (8) equals to zero. (d) Domain pattern described by Eq. (6). Unless otherwise specified, the pump Gaussian beam is located centrally at the position $x_{0}=-D \Lambda / 2$.

is proportional to the Fourier transform of $g(\mathbf{r}) C(\mathbf{r})$, where $g(\mathbf{r})$ is a normalized and dimensionless function that represents the space dependence of the nonlinear coefficient $\chi^{(2)}$, and $C(\mathbf{r})$ is an effective interaction area function. For traditional QPM interactions with the fundamental beam propagating in a plane perpendicular to the domain walls, as shown in Fig. 1(a), the function $C(\mathbf{r})$ is mainly defined by the crystal physical size [23]. Therefore, the beam width of the fundamental wave does not affect greatly the SHG via traditional QPM. However, the situation is quite different in Cerenkov-type SHG. With the fundamental beam propagating along the domain walls, as shown in Fig. 1(b), only those domains that are illuminated by the beam contribute to the nonlinear optical interaction. That is to say, the effective interaction area for the Cerenkov SHG is defined by the beam width of the fundamental wave, which consequently significantly affects the SH emission.

In order to reveal the physical features of Čerenkov $\mathrm{SH}$ emission and to understand better the role of the beam width on the radiation, in what follows we will solve analytically the wave functions for SHG. For simplicity, we consider the case of one-dimensional (1D) periodically poled crystal. However, the results shown here can be easily generalized to a twodimensional scheme and other second-order nonlinear processes, e.g., sum frequency generation and difference frequency generation. We assume that a Gaussian wave at frequency $\omega$ propagates along the domain walls ( $z$ axis). This wave generates a SH wave owing to the second-order susceptibility of the material. We assume that the fundamental frequency is linearly polarized, and we concentrate only on an ordinary polarization of the generated $\mathrm{SH}$ wave. Then coupling between the two beams is given by the element $d_{22}$ of the nonlinear susceptibility tensor $\chi^{(2)}$, the sign of which is spatially dependent on $x$ in a $1 \mathrm{D}$ structure.

Let us write the relevant amplitude of the $\mathrm{SH}$ wave as

$$
\mathbf{E}_{2 \omega}(x, z, t)=A_{2}(x, z) e^{i\left(2 \omega t-\rightarrow k_{2} \cdot \rightarrow z\right)} .
$$

We assume that the nonlinear conversion efficiency is low, hence the pump amplitude can be assumed constant throughout the entire interaction length (undepleted pump approximation). We further assume that the slowly varying envelope approximation applies for the SH wave. Under these assumptions, the evolution of the amplitude of the SH wave in the crystal can be described by the following paraxial equation:

$$
\left(\partial / \partial z+\frac{i}{2 k_{2}} \partial^{2} / \partial x^{2}\right) A_{2}(x, z)=-i g(x) \beta_{2} I_{1} F_{1}^{2}(x) e^{i \Delta k z},
$$

where $\Delta k=k_{2}-2 k_{1}$ is the mismatch of the wave vectors between the fundamental $\left(k_{1}\right)$ and the $\mathrm{SH}$ wave $\left(k_{2}\right), I_{1}$ is the maximum pump intensity at the center of the fundamental beam, $F_{1}(x)$ is the transverse distribution of the fundamental field and, for a Gaussian beam, it reads $F_{1}(x)=e^{-\left(x-x_{0}\right)^{2} / a^{2}}$, with $a$ being the beam width and $x_{0}$ denoting the center position of the beam, $\beta_{2}=k_{2} \chi^{(2)} /\left(2 n_{2}^{2}\right)$ is the nonlinear coupling coefficient, and $g(x)$ is the function characterizing the distribution of the second-order nonlinear susceptibility.

To solve Eq. (2), we change from the amplitude $A(x, z)$ to the Fourier spectrum:

$$
A_{2}\left(\kappa_{x}, z\right)=\int A_{2}(x, z) e^{i \kappa_{x} x} \mathrm{~d} x
$$

Here and below, we omit the limits of integration that is performed from $-\infty$ to $+\infty$. Then Eq. (2) takes the form

$$
\left(\partial / \partial z-i \kappa_{x}^{2} / 2 k_{2}\right) A_{2}\left(\kappa_{x}, z\right)=\Gamma \int g(x) F_{1}^{2}(x) e^{i \kappa_{x} x} e^{i \Delta k z} \mathrm{~d} x
$$

where $\Gamma=-i \beta_{2} I_{1}$. By solving this equation, the amplitude of the $\mathrm{SH}$ field can be expressed as

$$
\begin{aligned}
A_{2}\left(\kappa_{x}, z\right)= & \Gamma z e^{i z\left(\Delta k+\kappa_{x}^{2} / 2 k_{2}\right) / 2} \\
& \times \operatorname{sinc}\left[z\left(\Delta k-\kappa_{x}^{2} / 2 k_{2}\right) / 2\right] \int g(x) F_{1}^{2}(x) e^{i \kappa_{x} x} \mathrm{~d} x,
\end{aligned}
$$

with $\operatorname{sinc}(x)=\sin (x) / x$ and $\operatorname{sinc}(0)=1$.

For 1D periodic modulation of $\chi^{(2)}$, the function $g(x)$ can be expressed as the following Fourier series:

$$
g(x)=\sum_{m=0, \pm 1, \pm 2, \ldots} g_{m} e^{i m G_{0} x}
$$

Here $G_{0}=2 \pi / \Lambda$ is the primary reciprocal lattice vector $(\Lambda$ is the $\chi^{(2)}$ modulation period), the coefficients $g_{m}(m \neq 0)=$ $2 \sin (\pi m D) /(\pi m)$ and $g_{0}=2 D-1$ with $D$ being the duty cycle defined by the ratio of the length of the positive domains to the period of the $\chi^{(2)}$ structure. Then the integral in Eq. (5) can be evaluated as

$$
\begin{aligned}
\int g(x) F_{1}^{2}(x) e^{i \kappa_{x} x} \mathrm{~d} x= & a(\pi / 2)^{1 / 2} \\
& \times \sum_{m=0, \pm 1, \pm 2, \ldots} g_{m} e^{-a^{2}\left(m G_{0}+\kappa_{x}\right)^{2} / 8} e^{i\left(m G_{0}+\kappa_{x}\right) x_{0}} .
\end{aligned}
$$


By substituting Eq. (7) into Eq. (5) and considering the spectral density $S_{2}\left(\kappa_{x}, z\right)=\left|A_{2}\left(\kappa_{x}, z\right)\right|^{2}$ of the $\mathrm{SH}$, we obtain

$$
\begin{aligned}
S_{2}\left(\kappa_{x}, z\right)= & \pi a^{2} z^{2} \Gamma^{2} / 2 \times\left\{\operatorname{sinc}\left[z\left(\Delta k-\kappa_{x}^{2} / 2 k_{2}\right) / 2\right]\right\}^{2} \\
& \times\left(\sum_{m=0, \pm 1, \pm 2, \ldots} g_{m} e^{-a^{2}\left(m G_{0}+\kappa_{x}\right)^{2} / 8} e^{i m x_{0} G_{0}}\right)^{2} .
\end{aligned}
$$

From Eqs. (5) and (8), one finds that, when the sinc function equals to $\overline{1}$, i.e., for

$$
\Delta k-\kappa_{x}^{2} / 2 k_{2}=0,
$$

the generated intensity of the $\mathrm{SH} S_{2}\left(\kappa_{x}, z\right)$ is a quadratic function of the propagation distance $z$. The relation Eq. (9) represents the fulfillment of the longitudinal phase-matching condition. In fact, the condition of $\Delta k-\kappa_{x}^{2} / 2 k_{2}=0$ evolves into $k_{2}^{2}-\kappa_{x}^{2}-\left(2 k_{1}\right)^{2}=0$, taking into account $\left[1-\left(\kappa_{x} / k_{2}\right)\right]^{1 / 2} \approx$ $1-\kappa_{x} / 2 k_{2}$ provided $\kappa_{x}^{2} \ll k_{2}^{2}$. In Fig. $\underline{1(\mathrm{c})}$, we illustrate the corresponding phase-matching triangle constructed by the vectors $k_{2}, 2 k_{1}$, and $\kappa_{x}$. It is clear that, in this case, these three vectors form a right-angle triangle. Then we have relation $k_{2} \cos \theta=2 k_{1}$, which is exactly the well-known phasematching condition for the Čerenkov SHG, with $\theta$ being the angle between the fundamental and the $\mathrm{SH}$ waves.

\section{RESULTS AND DISCUSSION}

Now we present the results for the Čerenkov-type SHG in a 1D periodically poled ferroelectric crystal with the fundamental beam propagating along domain walls, as schematically shown in Fig. 1(b). We choose the $\mathrm{LiNbO}_{3}$ crystal as the nonlinear medium. Its nonlinear coefficient $d_{22}=1.1 \mathrm{pm} / \mathrm{V}$ [24], and its ordinary refractive index is given by the corresponding Sellmeier formula [25]. The crystal length is assumed to be $500 \mu \mathrm{m}$ and the poling period is set to $\Lambda=9 \mu \mathrm{m}$. The duty cycle is set as $D=0.5319$. Unless otherwise specified, the pump Gaussian beam is located centrally at the position of $x_{0}=-D \Lambda / 2$, i.e., it coincides exactly with one of the domain walls [see Fig. $\underline{1(\mathrm{~d})}$ ].

\section{A. Angular Distribution of Čerenkov SH Radiation and its Dependence on Propagation Length}

In Fig. 2 we show the dependence of the Čerenkov SH emission as a function of the internal emission angle. Here we depict results for fundamental wavelength $\lambda_{1}=1.108 \mu \mathrm{m}$, which is representative for the whole investigated frequency range. As shown in Fig. 2(a), the SH exhibits a symmetric angular distribution with respect to the propagation direction of the fundamental beam. The corresponding emission angles of two main peaks in Fig. $2(\mathrm{a})$ are $\pm 15.44^{\circ}\left(\kappa_{x}=\right.$ $\left.\pm 6.984 \mu \mathrm{m}^{-1}\right)$. They are in excellent agreement with the angles derived directly from the well-known Čerenkov relation $\theta_{C}=\cos ^{-1}\left(n_{1} / n_{2}\right)=15.439^{\circ}$, with $n_{1}$ and $n_{2}$ being the refractive indices of the fundamental and the $\mathrm{SH}$ waves, respectively.

In Fig. 2(b) we plot the dependence of the Čerenkov SH radiation on the propagation distance of the fundamental wave. It can be seen from Eq. (8) that the Čerenkov SH radiation, which is defined by the longitudinal phase-matching condition only, is coherently growing through the sample like a fully phase-matched process. This behavior agrees well with that obtained previously using the split-step beam propagation method [10].

\section{B. Wavelength Tuning with Fundamental Beams of Different Widths}

We now consider the dependence of the Čerenkov SH emission on the wavelength of the fundamental wave. It is seen from Eq. (8) that varying the wavelength of the fundamental beam leads to the generation of the Cerenkov SH at different spatial frequency $\kappa_{x}$, which causes variations of the emission angle as well as the output intensity of the SH signal. In the left column of Fig. 3, we show the wavelength response, i.e., the value of $S_{2}\left(\kappa_{x C}, z=L\right)$ of the Čerenkov $\mathrm{SH}$ generated by the fundamental Gaussian wave with different beam widths $(a)$. Here $\kappa_{x C}$ denotes the value of the spatial frequency $\kappa_{x}$ corresponding to Čerenkov angle. In these simulations, we assumed that the peak intensity of the fundamental beam was kept constant.

It is very interesting to see that, depending on the value of $a$, the wavelength tuning curves show quite different behaviors. When we use a relatively wide beam, e.g., $a=60 \mu \mathrm{m}$, the Čerenkov signal shows a series of sharp peaks as the wavelength varies [see Fig. 3(a)]. At these peak wavelengths the intensity of the emitted signal is quite high (e.g., at $\lambda_{1}=1.108 \mu \mathrm{m}$, marked by the red line in the left column of Fig. 3), while at the other positions it falls dramatically (e.g., at $\lambda_{1}=1.038 \mu \mathrm{m}$, marked by the green line). When the beam width is reduced to $10 \mu \mathrm{m}$, as shown in Fig. 3(b), the wavelength tuning peeks of the Čerenkov SH become broader. Moreover, while the previously (i.e., for $a=60 \mu \mathrm{m}$ ) strongest peaks decrease, the emission at the wavelengths which previously led to weak signal now significantly increases. When the beam width is reduced even further to $a=5 \mu \mathrm{m}$, the SH
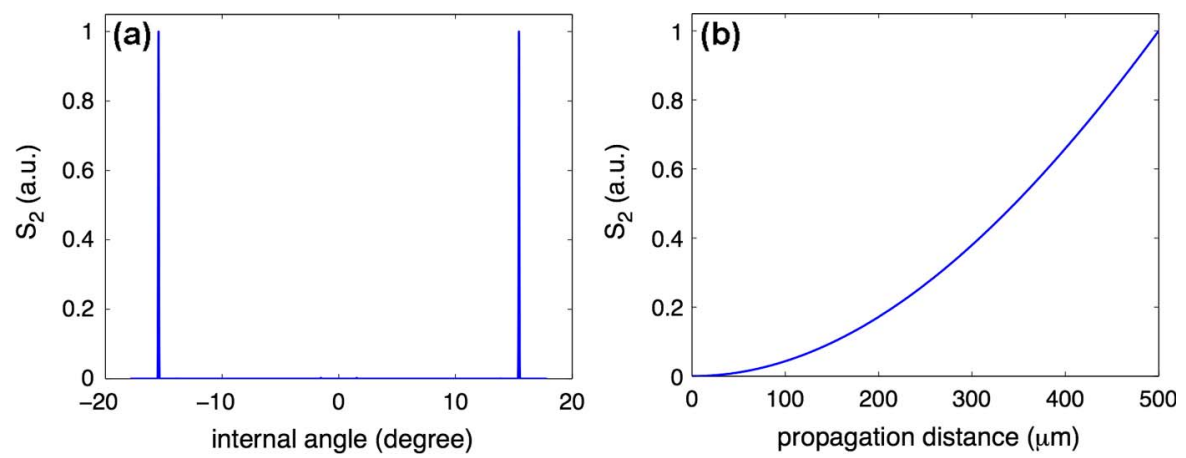

Fig. 2. (Color online) (a) Angular distribution of Čerenkov SHG signal for the fundamental wavelength of $1.108 \mu \mathrm{m}$. (b) Typical dependence of the intensity of the Čerenkov SH on the propagation length $\left(\kappa_{x}=6.984 \mu \mathrm{m}^{-1}\right)$. 

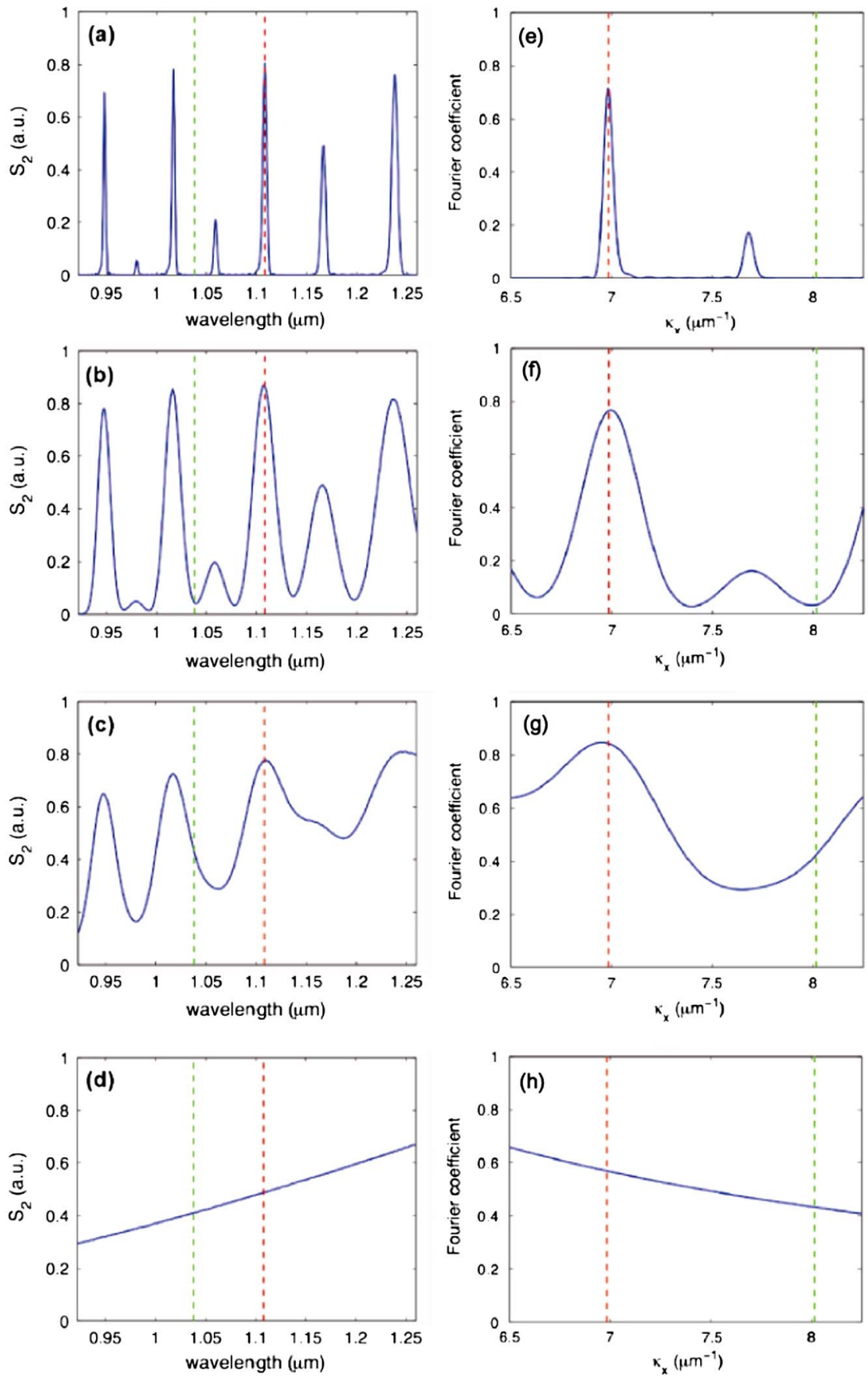

Fig. 3. (Color online) Left column: spectral response of the Čerenkov SHG for different beam widths. Right column: spatial Fourier spectrum of the Čerenkov emission. From top to bottom, the beam widths of the fundamental wave are $60,10,5$, and $2 \mu \mathrm{m}$. The plots are normalized to their individual maximum value. Vertical green and red lines indicate two particular wavelengths of the fundamental wave, $\lambda_{1}=1.038 \mu \mathrm{m}$ and $\lambda_{1}=1.108 \mu \mathrm{m}$, respectively.

peaks become so broad that they start overlapping. As a result, the wavelength response broadens. Finally, when the width of the fundamental beam becomes so narrow that it covers only a single domain wall (e.g., $a=2 \mu \mathrm{m}$ ), all SH peaks disappear and the Čerenkov intensity exhibits monotonic dependence on wavelength.

The dependence of the wavelength responses of the Čerenkov SH on the fundamental beam width can be understood by considering the corresponding properties in Fourier space. It follows from Eq. (5) that the intensity of the Čerenkov signal is determined by the Fourier transform of the product of the fundamental Gaussian beam $F_{1}^{2}(x)$ and the $g(x)$ function characterizing the spatial modulation of the sign of $\chi^{(2)}$. As demonstrated by Eq. (8), this Fourier transform function represents a set of Gaussian components with the width $2 / a$, the spacing between which equals $G_{0}=2 \pi / \Lambda$. Once a periodic $\chi^{(2)}$ crystal is produced, its period of modulation is fixed. This means that the spacing between two Gaussian components keeps constant no matter how wide is the fundamental beam. However, the widths of these components increase 
inversely with the beam width $a$, i.e., the smaller $a$ is, the wider the components become. In the right column of Fig. 3 we plot the form of this Fourier transform for different beam widths. The Fourier frequencies corresponding to the Čerenkov SHG at $\lambda_{1}=1.108 \mu \mathrm{m}$ and $\lambda_{1}=1.038 \mu \mathrm{m}$ are denoted by the red and green lines, respectively. When the beam width satisfies $a \gg 2 \Lambda / \pi$, i.e., the width of these components is much less than the spacing, a set of periodic peaks appear in the Fourier space and there is no overlapping between the two closest components [see Fig. 3(e), $a=60 \mu \mathrm{m}$ ]. Accordingly, the Cerenkov SH is at its intensity peaks when coinciding with those Fourier spectrum peaks. When the beam width is reduced to satisfy the condition $a \sim 2 \Lambda / \pi$, the Fourier components become broad and the closest components start overlapping. As a result, the Čerenkov SH shows a much wider tuning response [see Figs. 3(b) and (c), $a=10$ and $5 \mu \mathrm{m}]$. When $a \ll 2 \Lambda / \pi$, the widths of the Fourier components are already much broader than the spacing between them. As a result, the Fourier spectrum becomes a flat line. In this case, the Čerenkov SH intensity slightly increases with the wavelength. Being determined by the refractive index of $\mathrm{LiNbO}_{3}$ crystal, the generation of the Čerenkov $\mathrm{SH}$ at a longer fundamental wavelength involves a smaller magnitude of $\kappa_{x}$ and, hence, a larger Fourier coefficient [see Fig. 3(h)].

While the graphs shown in Fig. 3 display results obtained using the assumption of constant maximal intensity of the fundamental beam $\left(I_{1}\right)$, the same wavelength tuning response is preserved for the case of constant pump power of the fundamental beam. The narrower fundamental beam always leads to a broader bandwidth of the SH emission. It should be noted that, although in both cases the wavelength tuning response is the same, the absolute value of the emitted SH signal varies differently with the beam width. We will address this issue in the next section.

\section{Dependence of Čerenkov SH Emission on the Width of the Fundamental Beam}

We calculate the variation of the Čerenkov emission with the beam width of the fundamental wave at different wavelengths. The results for the SHG at $\lambda_{1}=1.108 \mu \mathrm{m}$ and $\lambda_{1}=1.038 \mu \mathrm{m}$ are displayed in Fig. 4, and are representative for the two kinds of typical results obtained in the whole examined wavelength range. To make the comparison more clearly, we perform two sets of calculations: first, we kept the peak intensity of the fundamental wave constant while varying the beam size; second, we kept the pump power constant. We see that the SH emission is dramatically different for different wavelengths. With constant peak intensity of the fundamental beam, while the SH signal grows quadratically with the beam width, as shown in Fig. 4(a) for $\lambda_{1}=1.108 \mu \mathrm{m}$, it quickly drops to zero in the case of $\lambda_{1}=1.038 \mu \mathrm{m}$, as shown in Fig. $4(\mathrm{~b})$. For the case of constant pump power, the SH signal drops quickly at both wavelengths, but it finally saturates at a higher energy level for $\lambda_{1}=1.108 \mu \mathrm{m}$ than that of $\lambda_{1}=$ $1.038 \mu \mathrm{m}$ [see Figs. $\underline{4(\mathrm{c})}$ and $\underline{(\mathrm{d})}$ ].

The different responses of the Čerenkov SH emission to the beam width can be understood by considering the form of Eq. (8). According to this equation, for constant peak intensity of the fundamental wave, the beam width affects the Čerenkov SH signal via two contributions. First, the spectral density $S_{2}$ is directly proportional to $a^{2}$. Second, the beam width also appears in the sum over all Fourier components of the nonlinearity modulation. For certain wavelengths (e.g., $\lambda_{1}=$ $1.108 \mu \mathrm{m}$ ), the argument of the exponents in the sum is zero,
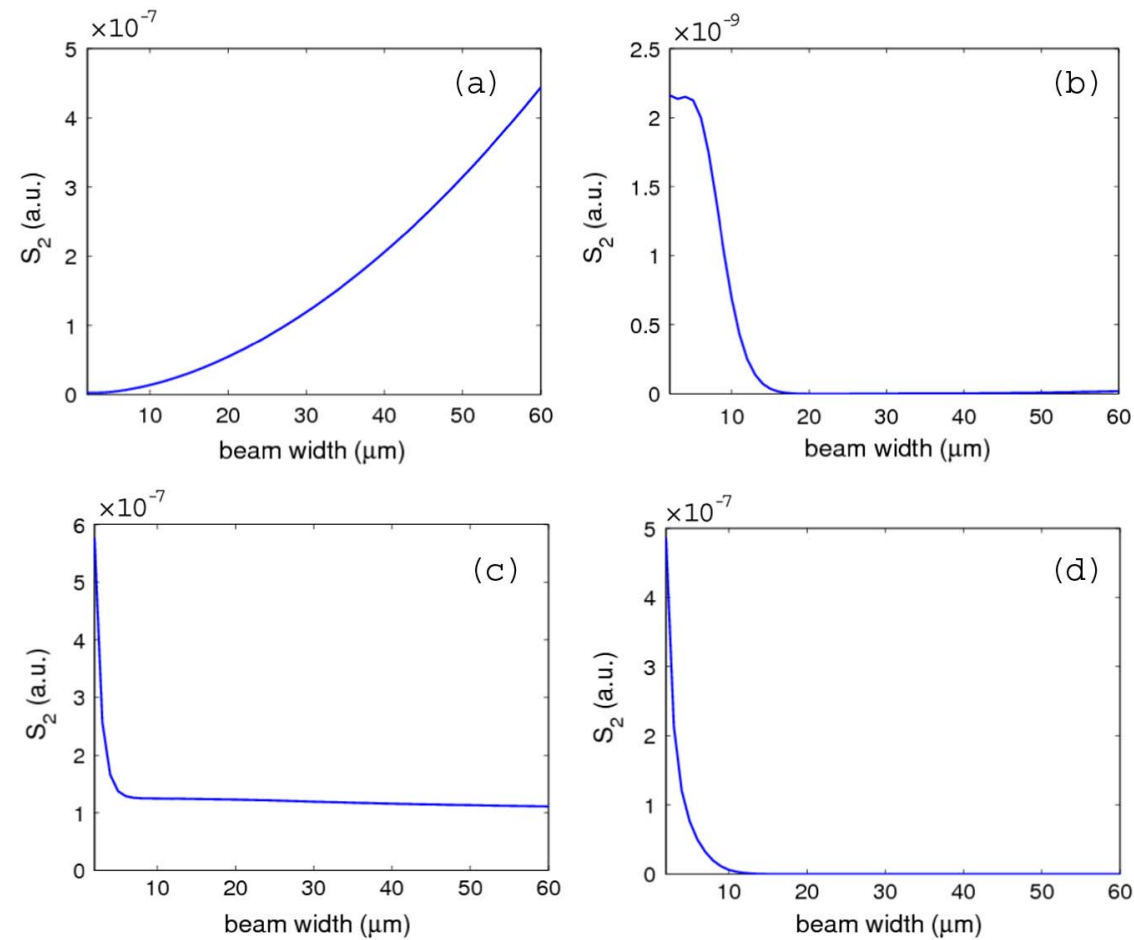

Fig. 4. (Color online) Čerenkov SH signal versus beam width for different wavelengths of the fundamental beam. (a) $\lambda=1.108 \mu \mathrm{m}$ and (b) $\lambda=1.038 \mu \mathrm{m}$ with the peak intensity of the fundamental wave being kept constant in both cases. (c) $\lambda=1.108 \mu \mathrm{m}$ and (d) $\lambda=1.038 \mu \mathrm{m}$ with constant pump power of the fundamental wave. $\kappa_{x}=6.984 \mu \mathrm{m}^{-1}$ for $\lambda=1.108 \mu \mathrm{m}$ and $\kappa_{x}=8.016 \mu \mathrm{m}^{-1}$ for $\lambda=1.038 \mu \mathrm{m}$. 


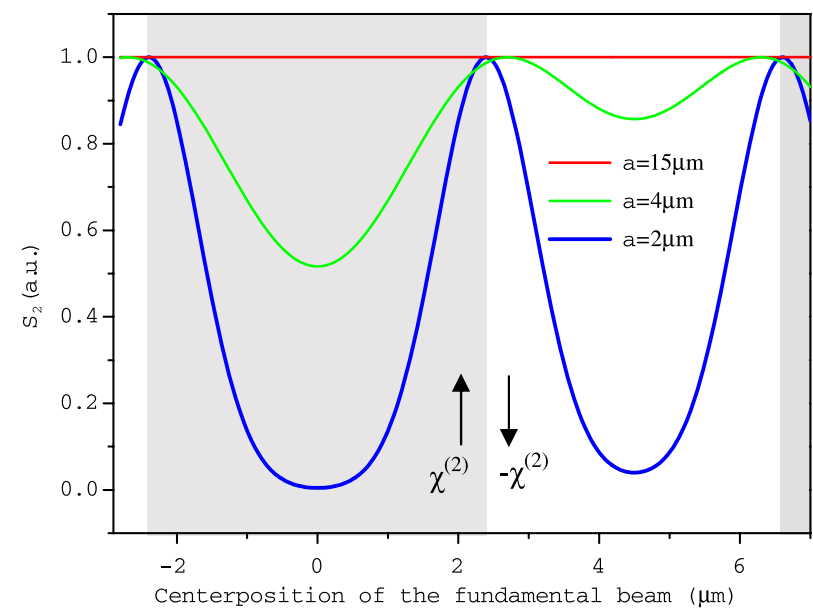

Fig. 5. (Color online) Čerenkov SH emission calculated when the fundamental beam is located at different positions $(\lambda=1.108 \mu \mathrm{m}$ and $\kappa_{x}=6.984 \mu \mathrm{m}^{-1}$ ). The blocks in gray and white represent the positive and reversed domains, respectively. It is clear that, in the case of a narrow beam, the Čerenkov SH is stronger when the beam is located exactly at the domain wall.

i.e., $m G_{0}+\kappa_{x}=0$. When this happens, the sum over the Fourier components does not depend on $a$ and, consequently, the SH spectral density $S_{2}$ is directly proportional to $a^{2}$. At the other wavelengths (e.g., $\lambda_{1}=1.038 \mu \mathrm{m}$ ), as $m G_{0}+\kappa_{x} \neq 0$, the Čerenkov harmonic intensity is determined by the product of $a^{2}$ and the sum of Gaussians. As the latter decays fast with beam width, the total intensity reaches maximum for small $a$ and then sharply decreases for larger beam width. The case of Čerenkov SHG with constant pump power can be explained in the same way. The only difference is that, in this case, the pump peak intensity $I_{1}$ decreases with the beam width and, hence, leads to weaker SH signals [see Figs. $\underline{4(\mathrm{c})}$ and (d)].

\section{Effect of the Center Position of the Fundamental Gaussian Beam}

Here we would like to point out that, in the case of Čerenkov SHG by a fundamental wave of very small beam size (compared to domain width), we also need to consider the effect of the center position of the fundamental beam. In Fig. $\underline{5}$ we show the Čerenkov SH intensity recorded as the position of the fundamental beam is scanned over a full period of domain structure. The beam width is set as $a=2,4$, and $15 \mu \mathrm{m}$, respectively, and the wavelength is $\lambda_{1}=1.108 \mu \mathrm{m}$. For a better and easier understanding of this result, we also plot the corresponding domain structure (the positive domains are marked by gray shading) in this figure. It can be seen that, with a narrow fundamental beam (e.g., $a=2$ and $4 \mu \mathrm{m}$ ), the Čerenkov emission is much stronger when the center of the fundamental beam is positioned exactly on or close to the domain wall. The SH becomes rather weak when the fundamental beam is moved away from the boundary into a homogeneous domains region. This enhancement of Čerenkov emission by the domain walls agrees quite well with our previous experimental results [20]. When a wider fundamental beam is used (e.g., $a=15 \mu \mathrm{m}$ ), the center position of the beam does not affect the Čerenkov SH intensity significantly. This is because, in this case, the fundamental beam covers almost the same number of domain walls no matter where it is located.

\section{CONCLUSION}

We studied analytically the Čerenkov harmonic generation by a Gaussian wave in a periodically poled $\mathrm{LiNbO}_{3}$ crystal. In particular, we considered the dependence of the harmonic radiation on the beam width of the fundamental wave. We found that the Čerenkov harmonic produced by the fundamental wave with a small beam width has a broader wavelength tuning response than that formed by a wider beam. We also demonstrated that, for a very narrow fundamental beam, the efficiency of the Čerenkov emission critically depends on the beam position with respect to the domain wall. These properties can be employed to optimize the wavelength response and the efficiency of generation of the Čerenkov SH signal in periodically poled structures. They are also important for utilizing the Cerenkov interaction in applications for high resolution nonlinear microscopy and novel light sources, including terahertz radiation.

\section{ACKNOWLEDGMENTS}

This work was supported by the Australian Research Council.

\section{REFERENCES}

1. P. A. Franken, A. E. Hill, C. W. Peters, and G. Weinreich, "Generation of optical harmonics," Phys. Rev. Lett. 7, 118-119 (1961).

2. R. C. Miller, "Optical haromonic generation in single crystal $\mathrm{BaTiO}_{3}$," Phys. Rev. 134, A1313-A1319 (1964).

3. V. G. Dmitriev, G. G. Gurzadyan, and D. N. Nikogosyan, Handbook of Nonlinear Optical Crystals (Springer-Verlag, 1991).

4. J. A. Amstrong, N. Bloembergen, J. Ducuing, and P. S. Pershan, "Interactions between light waves in a nonlinear dielectric," Phys. Rev. 127, 1918-1939 (1962).

5. S. Zhu, Y. Y. Zhu, and N. B. Ming, "Quasi-phase-matched thirdharmonic generation in a quasi-periodic optical superlattice," Science 278, 843-846 (1997).

6. V. Berger, "Nonlinear photonic crystals," Phys. Rev. Lett. 81, 4136-4139 (1998).

7. N. G. R. Broderick, G. W. Ross, H. L. Offerhaus, D. J. Richardson, and D. C. Hanna, "Hexagonally poled lithium niobate: a twodimensional nonlinear photonic crystal," Phys. Rev. Lett. 84, 4345-4348 (2000).

8. A. Arie and N. Voloch-Bloch, "Periodic, quasi-periodic, and random quadratic nonlinear photonic crystals," Laser Photon. Rev. 4, 355-373 (2010).

9. Y. Sheng, J. Dou, B. Ma, B. Cheng, and D. Zhang, "Broadband efficient second harmonic generation in media with a shortrange order," Appl. Phys. Lett. 91, 011101 (2007).

10. S. M. Saltiel, Y. Sheng, N. Voloch-Bloch, D. N. Neshev, W. Krolikowski, A. Arie, K. Koyonov, and Y. S. Kivshar, "Čerenkov-type second harmonic generation in two-dimensional nonlinear photonic structures," IEEE J. Quantum Electron. 45, 1465-1472 (2009).

11. Y. Zhang, Z. D. Gao, Z. Qi, S. N. Zhu, and N. B. Ming, "Nonlinear Čerenkov radiation in nonlinear photonic crystal waveguides," Phys. Rev. Lett. 100, 163904 (2008).

12. Y. Sheng, S. M. Saltiel, W. Krolikowski, A. Arie, K. Koynov, and Y. S. Kivshar, "Čerenkov-type second-harmonic generation with fundamental beams of different polarizations," Opt. Lett. 35, 1317-1319 (2010).

13. W. Wang, Y. Sheng, Y. Kong, A. Arie, and W. Krolikowski, "Multiple Čerenkov second-harmonic waves in a two-dimensional nonlinear photonic structure," Opt. Lett. 35, 3790-3792 (2010)

14. I. V. Shutov, I. A. Ozheredov, A. V. Shumitski, and A. S. Chirkin, "Second harmonic generation by femtosecond laser pulse in the Laue scheme," Opt. Spectrosc. 105, 79-84 (2008).

15. P. Molina, M. O. Ramirez, B. J. Garcia, and L. E. Bausa, "Directional dependence of the second harmonic response in twodimensional nonlinear photonic crystals," Appl. Phys. Lett. 96, 261111 (2010).

16. J. V. Jelley, Cerenkov Radiation (Pergamon, 1958). 
17. A. Zembrod, H. Puell, and J. Giordmaine, "Surface radiation from non-linear optical polarisation," Opt. Quantum Electron. 1, 64-66 (1969)

18. A. A. Kaminskii, H. Nishioka, K. Ueda, W. Odajima, M. Tateno, K. Sasaki, and A. V. Butashin, "Second-harmonic generation with Cherenkov-type phase matching in a bulk nonlinear $\mathrm{LaBGeO}_{5}$ crystal," Quantum Electron. 26, 381-382 (1996).

19. V. Vacaitis, "Cherenkov-type phase matching in bulk KDP crystal," Opt. Commun. 209, 485-490 (2002).

20. Y. Sheng, A. Best, H. Butt, W. Krolikowski, A. Arie, and K. Koynov, "Three-dimensional ferroelectric domain visualization by Čerenkov-type second harmonic generation," Opt. Express 18, 16539-16545 (2010).

21. A. Fragemann, V. Pasiskevicus, and F. Laurell, "Second-order nonlinearites in the domain walls of peri- odically poled $\mathrm{KTiOPO}_{4}, "$ Appl. Phys. Lett. 85, 375-377 (2004).

22. X. Deng and X. Chen, "Domain wall characterization in ferroelectrics by using localized nonlinearities," Opt. Express 18, 15597-15602 (2010).

23. A. Arie, N. Habshoosh, and A. Bahabad, "Quasi phase matching in two-dimensional nonlinear photonic crystals," Opt. Quantum Electron. 39, 361-375 (2007).

24. A. Ganany, A. Arie, and S. M. Saltiel, "Quasi-phase matching in $\mathrm{LiNbO}_{3}$ using nonlinear coefficients in the $X Y$ plane," Appl. Phys. B 85, 97-100 (2006).

25. G. J. Edwards and M. Lawrence, "A temperature-dependent dispersion equation for congruently grown lithium niobate," Opt. Quantum Electron. 16, 373-375 (1984). 